\title{
Standard model from a supergravity model with a naturally small cosmological constant
}

\author{
Shing Yan Li, ${ }^{a}$ Yu-Cheng Qiu ${ }^{b}$ and S.-H. Henry Tye ${ }^{b, c}$ \\ ${ }^{a}$ Center for Theoretical Physics, Department of Physics, \\ Massachusetts Institute of Technology, Cambridge, MA 02139, U.S.A. \\ ${ }^{b}$ Department of Physics and Jockey Club Institute for Advanced Study, \\ The Hong Kong University of Science and Technology, Hong Kong, S.A.R. China \\ ${ }^{c}$ Department of Physics, Cornell University, \\ Ithaca, NY 14853, U.S.A. \\ E-mail: sykobeli@mit.edu, yqiuai@connect.ust.hk, iastye@ust.hk, \\ sht5@cornell.edu
}

ABSTRACT: Guided by the naturalness criterion for an exponentially small cosmological constant, we present a string theory motivated 4-dimensional $\mathcal{N}=1$ non-linear supergravity model (or its linear version with a nilpotent superfield) with spontaneous supersymmetry breaking. The model encompasses the minimal supersymmetric standard model, the racetrack Kähler uplift, and the KKLT anti-D3-branes, and use the nilpotent superfield to project out the undesirable interaction terms as well as the unwanted degrees of freedom to end up with the standard model (not the supersymmetric version) of strong and electroweak interactions.

KEYWORDS: Strings and branes phenomenology

ARXIV EPrint: 2010.10089 


\section{Contents}

1 Introduction 1

2 Model 3

3 Properties 4

4 Higgs sector $\quad 5$

$\begin{array}{llr}5 & \text { Summary } & 8\end{array}$

$\begin{array}{llr}6 & \text { Comments } & 8\end{array}$

\section{Introduction}

The dark energy, or the observed cosmological constant $\Lambda_{\text {obs }}$, poses a puzzle in fundamental physics. Its smallness with respect to the (reduced) Planck mass $\left(M_{\mathrm{Pl}}=2.4 \times 10^{18} \mathrm{GeV}\right)$, $\Lambda_{\mathrm{obs}} \simeq 10^{-120} M_{\mathrm{Pl}}^{4}$, needs to be understood. Since $\Lambda$ is calculable in string theory, one is led to search for a reason within string theory. Hopefully, understanding its smallness will also reveal other structures in nature. Here is an attempt along this direction.

It has been shown that the string theory motivated racetrack Kähler uplift (RKU) model allows an exponentially small cosmological constant $\Lambda$ [1]. Sweeping over a patch of the string landscape (i.e., scanning over the parameters of the model), one finds that the probability distribution $P(\Lambda) \propto \Lambda^{-1+k}$ for $\Lambda \rightarrow 0^{+}$, where $1 \gg k>0$. An exponentially small positive $\Lambda$ (e.g., the median value) naturally follows from the divergent behavior of such a properly normalized $P(\Lambda)$ at $\Lambda \rightarrow 0^{+}$. In this RKU model, the value of the observed $\Lambda_{\text {obs }}$ implies the emergence of a new scale $\mathbf{m}$, which happens to be close to the electroweak scale, $\mathbf{m} \sim m_{\mathrm{EW}} \sim 10^{2} \mathrm{GeV}[2]$. The KKLT scenario [3] suggests that an anti-D3-(D3)brane can break supersymmetry (SUSY) and uplift the vacuum to a de Sitter (dS) vacuum. So one is led to include into the RKU model the contributions of the (warped) $\overline{\mathrm{D} 3}$-brane tension $m_{s}^{4}$ as well as the effective Higgs potential $V_{h}$ after spontaneous symmetry breaking (SSB). It is shown that their respective contributions must cancel to a high degree in order to maintain a naturally small $\Lambda$ in the RKU model [4],

$$
(\Delta V)_{\min }=\left|m_{s}^{4}+V_{h, \min }\right|<\Lambda,
$$

where the negative $V_{h, \min } \simeq-\mathcal{O}\left(m_{\mathrm{EW}}^{4}\right)$. (In fact, there is no meta-stable dS vacuum solution in the RKU model if $\Delta V>\Lambda$.) This cancellation must be automatic; otherwise, fine-tuning is re-introduced. In this paper, let us focus on this condition, which is now a part of the naturalness criterion. Since the Higgs potential $\left|V_{h, \min }\right|$ is many orders of 
magnitude larger than $\Lambda_{\text {obs }}$, one should expect $V_{h \text {,min }}$ to be canceled or screened by some effect without fine-tuning in any model with a naturally small $\Lambda$. In this paper, we show how this is achieved in a specific SUGRA model with $\overline{\mathrm{D} 3}$-brane(s).

Note that, in scanning over parameters of the model to obtain $P(\Lambda)$, we scan over all the parameters in $\Delta V(1.1)$ too. A naturally vanishingly small $(\Delta V)_{\text {min }}$ with parameters spanning over (reasonable) ranges imposes tight constraints in model building. Not surprisingly, this has strong implications on particle physics phenomenology.

The motivation of the model comes from string theory. In terms of the Planck mass scale, $m_{s} \simeq m_{\mathrm{EW}} \sim 10^{-16} M_{\mathrm{Pl}}$. In the brane world scenario with the warped flux compactification in Type IIB string theory [cf. [5-8]], this strongly suggests that both the standard model (SM) of strong and electroweak interactions and the $\overline{\mathrm{D} 3}$-brane must sit at the bottom (or close to the bottom) of the same warped throat. This further suggests that the SM particles are simply open string modes inside the $\overline{\mathrm{D} 3}$-brane (or a stack of $p \geq 5$ of them that span our 3-dimensional observable universe) [4], or more generally, inside a $\overline{\mathrm{D} 3} / \mathrm{D} 7$ system, with a set of intersecting D7-branes. Similar scenarios have been suggested earlier [9-14]. In this paper, the naturalness criterion (1.1) provides crucial guidance in developing the model.

It was suspected that SUSY breaking by (warped) $\overline{\mathrm{D} 3}$-brane(s) is spontaneous [15-17]. It turns out that this spontaneous SUSY breaking by an $\overline{\mathrm{D} 3}$-brane can be incorporated into a non-linear SUSY model [18], which can be cast as a linear SUSY model with a nilpotent chiral superfield $X$, where $X^{2}=0$ [19-23]. (This is analogous to a non-linear sigma model cast as a linear sigma model with a constraint.) To start the construction of the model, we assume that the minimal supersymmetric standard model (MSSM) [cf. [24]] emerges from such a $\overline{\mathrm{D} 3} / \mathrm{D} 7$ system.

Putting together the RKU model, the KKLT model and MSSM leads us to a 4dimensional $\mathcal{N}=1$ supergravity model, namely a minimal non-linear (or nilpotent) SUSY standard model (mNSSM). In MSSM, some of the terms in the Higgs potential contribute an electroweak scale (semi-positive) vacuum energy density which is many orders of magnitude bigger than $\Lambda_{\text {obs }}$. To satisfy (1.1), such terms have to be projected out, so the new resulting potential $\Delta V$ can vanish naturally after SSB. Our approach uses $X$ to impose constraints [25-27] on the particle spectrum as well as terms in the Higgs potential so that the model satisfies condition (1.1). As a result, the Higgsinos and half of the two Higgs doublets are projected out, and the final Higgs potential for all practical purposes is precisely that in the SM, with the SM Higgs doublet $h=\left(h^{0}, h^{-}\right)$,

$$
\Delta V=\left|m_{s}^{2}-\kappa h^{\dagger} h\right|^{2}
$$

where $(\Delta V)_{\min }=0$ after SSB, irrespective of the values of $m_{s}^{2}$ and the real positive $\kappa$. That is, the warped $\overline{\mathrm{D} 3}$-brane tension $m_{s}^{4}$ and the Higgs potential $V_{h \text {,min }}$ completely cancel each other to satisfy (1.1).

Note that the supposed SUSY breaking from $\overline{\mathrm{D} 3}$-brane(s) (in the KKLT scenario) is now completely screened by the Higgs potential. As a consequence, the SUSY breaking in the model comes entirely from the Kähler uplift, which is exponentially small. This implies that a SUSY pair have almost degenerate masses; since superpartners have not 
been observed experimentally, all remaining superpartners in MSSM must be removed, again employing $X$ to project them out. The final model is for all practical purposes precisely the SM of strong and electroweak interactions. However, the superpartners of the (closed) string modes (such as the graviton) that are not confined to the $\overline{\mathrm{D} 3}$-brane(s) are expected to remain.

\section{Model}

In the Wilsonian effective field theory approach, we write down a low energy 4-dimensional $\mathcal{N}=1$ supergravity model with parameters calibrated at the electroweak energy scale. Besides the contribution of the $\mathrm{SU}(3) \times \mathrm{SU}(2) \times \mathrm{U}(1)$ gauge supermultiplets, the model is specified by the Kähler potential $K$ and the superpotential $W$. (Bar on quantities implies complex conjugate.)

$$
\begin{aligned}
K & =-2 \ln \left[\left(T+\bar{T}-X \bar{X}-n_{u} H_{u}^{\dagger} H_{u}-n_{d} H_{d}^{\dagger} H_{d}+K_{\text {matter }}\right)^{3 / 2}+\frac{\xi}{2}\right], \\
W & =W_{0}\left(U_{i}, S\right)+W_{\mathrm{np}}(T)+\tilde{\mu} H_{u} H_{d}-X\left(\tilde{m}_{s}^{2}+\tilde{\gamma} H_{u} H_{d}\right)+W_{\text {matter }}, \\
W_{\mathrm{np}}(T) & =A e^{-a T}+B e^{-b T}, \quad \xi=-\frac{\zeta(3)}{4 \sqrt{2}(2 \pi)^{3}} \chi(M)(S+\bar{S})^{3 / 2}>0 .
\end{aligned}
$$

Here, $K_{\text {matter }}$ and $W_{\text {matter }}$ contain the quark and lepton superfield contributions in MSSM, including all the Yukawa couplings in $W_{\text {matter. }}$. The complex structure moduli $U_{i}$ and the dilaton $S$ are stabilized [3, 4, 28], thus $W_{0}$ can be treated as a constant, leaving only the Kähler modulus $T$ which measures the volume of the flux-compactified Calabi-Yau orientifold $\boldsymbol{M}$. The Kähler uplift is provided by $\xi$, a stringy $\alpha^{\prime 3}$-correction [29]; with multiple $U_{i}$, the Euler index of the manifold $\chi(M)<0$, so $\xi>0$. We expect $\xi \sim \mathcal{O}\left(10^{-2}\right)$ in the KU model [30-35]. The non-perturbative $W_{\mathrm{np}}$, which can come from gaugino condensation in D7-branes, provides the racetrack. Together, they form the RKU model when $T$ is being stabilized. In the brane world picture in string theory, $U_{i}, S, T$ and the graviton-gravitino pair are close string modes while the rest are open string modes inside the $\overline{\mathrm{D} 3}$-brane(s) that span our observable universe.

Here the two Higgs doublets $H_{u}$ and $H_{d}$ in MSSM are explicitly displayed, since only Higgs fields acquire vacuum expectation values (vev) in SM and contribute to $\Lambda$. We denote $H_{u} H_{d}=\left(H_{u}\right)_{i}\left(H_{d}\right)_{j} \epsilon^{i j}=H_{u}^{+} H_{d}^{-}-H_{u}^{0} H_{d}^{0} . n_{u}$ and $n_{d}$ are $\mathcal{G}(1)$ normalization constants. $\tilde{\gamma}$ describes the (dimensionless) coupling between $X$ and Higgs superfields. The scalar potential $V$ is given by two parts, namely the $F$-terms and the $D$-terms. Possible $D$-term contribution from the geometrical sector is ignored here. In units where $M_{\mathrm{Pl}}=1$,

$$
\begin{aligned}
V & =K_{I \bar{J}} F^{I} \bar{F}^{\bar{J}}-3 e^{K} W \bar{W}+V_{D} \\
& =V_{T}+V_{X}+V_{H, F}+V_{H, D}=V_{T}+\Delta V,
\end{aligned}
$$

where auxiliary field $F^{I}=e^{K / 2} K^{I \bar{J}} D_{\bar{J}} \bar{W}, D_{I}=\partial_{I}+K_{I}, K_{I}=\partial_{I} K, K^{I \bar{J}} K_{I \bar{J}}=1$ and

$$
\begin{aligned}
& V_{T}=e^{K} K^{T \bar{T}}\left|D_{T} W\right|^{2}-3 e^{K}|W|^{2}, \\
& V_{X}=K_{X} \bar{X}^{X} \bar{F}^{\bar{X}}+\left(K_{T} \bar{X}^{T} \bar{F}^{\bar{X}}+\text { c.c. }\right),
\end{aligned}
$$




$$
\begin{aligned}
& V_{H, F}=K_{H \bar{H}} F^{H} \bar{F}^{\bar{H}}+\left(K_{H \bar{I}} F^{H} \bar{F}^{\bar{I}}+\text { c.c. }\right), \\
& V_{H, D}=\sum_{a} \frac{1}{2} g_{a}^{2} D^{a 2} .
\end{aligned}
$$

In the RKU model where only $V_{T}$ is present, it has a no-scale structure when $\xi=0$ and a small positive $\Lambda$ emerges after $T$ is stabilized with $\xi$ turned on. $g_{a}$ is gauge coupling and $D^{a}$ is the corresponding Killing potential in supergravity language. Contributions (2.3b), (2.3c) and (2.3d) are all semi-positive squares. To minimize $V$ (to satisfy condition (1.1)), we need $\Delta V=V_{X}+V_{H, F}+V_{H, D}<\Lambda$, that is, for all practical purposes, each term should vanish by itself. To achieve that, we introduce several algebraic constraints $[26,27,36]$ to project out some undesired degrees of freedom as well as interactions; explicitly,

$$
\begin{aligned}
X^{2} & =0, \\
X \bar{H} & =\text { chiral }, \\
X\left[\left(H_{u}\right)_{i}-\epsilon_{i j}\left(\bar{H}_{d}\right)^{j}\right] & =0, \\
X Q_{i}=X L_{j}=X W_{\alpha} & =0 .
\end{aligned}
$$

In short, (2.1) together with (2.4) defines our mNSSM model. Here $X$ plays the role of a projection operator that removes field degrees of freedom and the constraints are off-shell conditions. The constraint (2.4b) (i.e., $\overline{\mathscr{D}}_{\dot{\alpha}}\left[X \bar{H}_{i}\right]=0$ ) is on every Higgs chiral superfield, and $\epsilon_{i j} \epsilon^{j k}=\delta_{i}^{k}$ in (2.4c). In (2.4d), index $i$ runs over all quark chiral superfields, the index $j$ runs over all lepton chiral superfields and index $\alpha$ runs over all the field strength chiral superfields of the vector superfields. In the context of string theory ( $\overline{\mathrm{D} 3}$-brane in an orientifold), constraint $(2.4 \mathrm{a})$ is discussed in refs. [22, 23], while constraints $(2.4 \mathrm{~b})$ and $(2.4 \mathrm{~d})$ are discussed in refs. [37, 38].

As we shall see, $V_{X}=0$ is automatic after SSB, while constraint (2.4b) eliminates the term $V_{H, F}$ and the constraint (2.4c) eliminates $V_{H, D}$ terms (note that both $V_{H, F}$ and $V_{H, D}$ are present in MSSM). The last set $(2.4 \mathrm{~d})$ of constraints project out the remaining R-parity odd degrees of freedom, i.e., the scalar quarks and scalar leptons as well as the gauginos in MSSM; as we shall see, this is necessary since SUSY breaking is negligibly small in the model.

\section{Properties}

The nilpotent superfield $X$ emerges from the presence of an $\overline{\mathrm{D} 3}$-brane [13, 21, 22]. The constraint $X^{2}=0(2.4 \mathrm{a})$ projects out the scalar degree of freedom of $X$. Expanded in supergravity variables $\Theta[39]$,

$$
X=x+\sqrt{2} \Theta G+\Theta^{2} F^{X} \text { and } x=G G / 2 F^{X},
$$

where $G_{\alpha}$ is the fermion which contributes to part of goldstino and $F^{X}$ is the auxiliary field. Since the expectation value $\langle G G\rangle=0, x$ and any field component in (2.3) that contains $G_{\alpha}$ (due to constraints (2.4)) will drop out in $V .{ }^{1}$ Therefore, only the $K_{X \bar{X}}$ term

\footnotetext{
${ }^{1}$ These constraints are operator identities, which means that these solution exists as long as $F^{X} \neq 0$. However, the classical value $\left\langle F^{X}\right\rangle$ can be zero after SSB.
} 
remains in $(2.3 \mathrm{~b})$ and we have

$$
V_{X}=\frac{\left|\tilde{m}_{s}^{2}+\tilde{\gamma} H_{u} H_{d}\right|^{2}}{3(T+\bar{T}+\cdots)^{2}} .
$$

Next, let us consider a quark chiral superfield $Q=q+\sqrt{2} \Theta \psi+\Theta^{2} F^{Q}$. The constraint $X Q=0(2.4 \mathrm{~d})$ yields $[26]$

$$
\begin{aligned}
Q & =\frac{\psi G}{F^{X}}-\frac{G^{2} F^{Q}}{2\left(F^{X}\right)^{2}}+\sqrt{2} \Theta \psi+\Theta^{2} F^{Q} \\
& =\sqrt{2}\left(\psi-\frac{F^{Q} G}{F^{X}}\right) \tilde{\Theta}+F^{Q} \tilde{\Theta}^{2},
\end{aligned}
$$

where $\tilde{\Theta}=\Theta+G / \sqrt{2} F^{X}$. Here the scalar quark is projected out. (Since $G_{\alpha}$ plays no role in the determination of $\Delta V$, we can consider a simpler $X=\theta^{2} F^{X}$ which satisfies $X^{2}=0$ [26]. Here $X Q=0$ implies $q F^{X}=0$ so $q$ is projected out.) The other constraints are similar.

\section{Higgs sector}

$V_{H, F}(2.3 \mathrm{c})$ in $V(2.2)$ will in general violate (1.1) without fine-tuning, so we like to remove it. Constraint (2.4b) on a Higgs superfield yields, in global SUSY [26],

$$
\begin{aligned}
H & =h+\sqrt{2} \theta \Psi_{H}+\theta^{2} F^{H}, \\
\Psi_{H} & =i \sigma^{\nu}\left(\frac{\bar{G}}{\bar{F}^{\bar{X}}}\right) \partial_{\nu} h, \\
F^{H} & =-\partial_{\mu}\left(\frac{\bar{G}}{\bar{F}^{\bar{X}}}\right) \bar{\sigma}^{\nu} \sigma^{\mu} \frac{\bar{G}}{\overline{F^{X}}} \partial_{\nu} h+\frac{1}{2\left(\overline{F^{X}}\right)^{2}} \bar{G}^{2} \partial^{2} h,
\end{aligned}
$$

where the corresponding expressions in SUGRA can be found in refs. [27]. We see that, not only $\Psi_{H}$ is removed, so is the corresponding auxiliary field $F^{H}$. Applying the constraint (2.4b) on every Higgs superfield, the auxilliary fields $F^{H}$ are removed, and (2.3c) yields (after setting the expectation value of $G_{\alpha}$ to zero)

$$
V_{H, F} \propto|\tilde{\mu}|^{2}\left(H_{u}^{\dagger} H_{u}+H_{d}^{\dagger} H_{d}\right)+\cdots \rightarrow 0 .
$$

This means that $\tilde{\mu} H_{u} H_{d}$ in $W(2.1)$ contributes to the scalar potential (of order $\left.\mathcal{O}\left(m_{\mathrm{EW}}^{6} / M_{\mathrm{Pl}}^{2}\right)\right)$ only in $V_{T}$, which is already included in the RKU model [4].

Now let us expand the whole scalar potential and focus on Higgs fields. Relevant and marginal terms are kept and higher order terms which is suppressed by $M_{\mathrm{Pl}}$ are ignored. Having in mind that $T+\bar{T} \sim \mathcal{O}\left(10^{3}\right)$ (so the string scale $M_{S} \sim 10^{15}-10^{16} \mathrm{GeV}$ ), we only keep terms proportional to $(T+\bar{T})^{-2}$. Due to the no-scale structure of the Kähler potential, Higgs fields receive field normalization according to their respective kinetic terms (after the $T$ stabilization),

$$
h_{u}=h_{u}\left(\frac{3 n_{u}}{T+\bar{T}}\right)^{1 / 2}, \quad h_{d}=h_{d}\left(\frac{3 n_{d}}{T+\bar{T}}\right)^{1 / 2}
$$


so that kinetic terms of $h_{u}$ and $h_{d}$ are in canonical form, which means that they are the observed Higgs fields in MSSM. The remaining dependence of $(T+\bar{T})$ in the potential can be absorbed into the parameters,

$$
m_{s}=\tilde{m}_{s}\left[3(T+\bar{T})^{2}\right]^{-1 / 4}, \quad \gamma=\tilde{\gamma}\left(27 n_{u} n_{d}\right)^{-1 / 2},
$$

where the $D$-term potential is the same as that in MSSM. There should be some $T$ dependence in $V_{H, D}$, which could be further absorbed into $n_{u}$ and $n_{d}$ after normalization of Higgs fields. We write down the potential for two Higgs doublets with all parameters rescaled to observed value properly,

$$
V_{2 h}=2 m_{s}^{2} \operatorname{Re}\left(\gamma h_{u} h_{d}\right)+\left|\gamma h_{u} h_{d}\right|^{2}+\frac{g^{2}+g^{\prime 2}}{8}\left(\frac{\left|h_{u}\right|^{2}}{n_{u}}-\frac{\left|h_{d}\right|^{2}}{n_{d}}\right)^{2}+\frac{g^{2}}{2}\left|\frac{h_{u}^{\dagger} h_{d}}{\sqrt{n_{u} n_{d}}}\right|^{2},
$$

where the first two terms come from $V_{X}$ and the last two terms from $V_{H, D} ; g$ and $g^{\prime}$ are the $\mathrm{SU}(2)$ and $\mathrm{U}(1)$ gauge couplings respectively, and $h_{u}=\left(h_{u}^{+}, h_{u}^{0}\right), h_{d}=\left(h_{d}^{0}, h_{d}^{-}\right)$as in MSSM. With the SU(2) symmetry, we choose SSB as $\left\langle h_{u}^{0}\right\rangle$ develops a vev, followed by $\left\langle h_{d}^{0}\right\rangle \neq 0$. Parameter $\gamma$ is a complex number in principle. It can be made real by rotating the complex fields $h_{u}^{0}$ and $h_{d}^{0}$, so that $\gamma>0$ and $\gamma\left\langle h_{u} h_{d}\right\rangle=-\gamma\left\langle h_{u}^{0}\right\rangle\left\langle h_{d}^{0}\right\rangle=-\gamma v_{u} v_{d}<0$.

After SSB, $v_{u} v_{d}=m_{s}^{2} / \gamma, v_{d}^{2}=m_{s}^{2} \sqrt{n_{d} / n_{u}} / \gamma$, so $v_{u}^{2}=m_{s}^{2} \sqrt{n_{u} / n_{d}} / \gamma$. Following MSSM, we introduce $\tan \beta=v_{u} / v_{d}$. It turns out that, at the minimum,

$$
\tan \beta=\sqrt{\frac{n_{u}}{n_{d}}} \rightarrow\left\{\begin{array}{l}
V_{H, D, \min }=0 \\
V_{2 h, \min }=-m_{s}^{4}
\end{array} \quad \rightarrow V_{X, \min }=0\right.
$$

so $(\Delta V)_{\min }=0$ and condition (1.1) is satisfied. Note that the result $(\Delta V)_{\min }=0$ is insensitive to the values of $m_{s}, \gamma, g^{2}, g^{\prime 2}, n_{u}, n_{d}$ and $\mu$.

If the $|\tilde{\mu}|^{2}$ term in $V_{H, F}(4.2)$ is present, as in MSSM, then $V_{2 h}$ (4.5) will have an additional term (setting $n_{u}=n_{d}=1$ and with a rescaled $\mu$ ): $V_{2 h} \rightarrow V_{2 h}+|\mu|^{2}\left(\left|h_{u}\right|^{2}+\left|h_{d}\right|^{2}\right)$. In this case $V_{2 h, \text { min }}=-\left(m_{s}^{2}-|\mu|^{2} / \gamma\right)^{2}$ yielding $\Delta V=m_{s}^{4}-\left(m_{s}^{2}-|\mu|^{2} / \gamma\right)^{2}>0$, so condition (1.1) is not satisfied except via fine-tuning. This is the reason we impose (2.4b) to obtain (4.2); as a consequence, Higgsinos are absent in this model. However, for a very small $W_{0}\left(U_{i}, S\right)$ (as expected), the observed $\Lambda_{\text {obs }}$ implies that $\left|\mu h_{u} h_{d}\right| \sim \mathbf{m}^{3} \sim(100 \mathrm{GeV})^{3}[2,4]$.

Although the above result (4.6) with $V_{H, D \text {, min }}=0$ is desirable, it is straightforward to check that the charged Higgs boson mass is the same as the $W$-boson mass, $m_{H^{ \pm}}^{2}=m_{W}^{2}=$ $\left(v_{u}^{2}+v_{d}^{2}\right) g^{2} / 2$, which is disastrous, as experiments show such $H^{ \pm}$do not exist. Fortunately, this problem can be easily solved by the constraint (2.4c), which projects out $V_{H, D}(2.3 \mathrm{~d})$ as well as half of the Higgs degrees of freedom.

Let us explain the choice of the particular linear combination in the constraint (2.4c). Similar to (3.3), a linear combination of the Higgs scalar fields is projected out. Suppose, instead of (2.4c), we impose $X\left[\left(H_{u}\right)_{i}-q \epsilon_{i j}\left(\bar{H}_{d}\right)^{j}\right]=0$, where $q$ is real but arbitrary. This projection gives a linear relation between observed (properly normalized according to (4.3)) Higgs fields,

$$
h_{u}^{+}=-q \sqrt{\frac{n_{u}}{n_{d}}} \bar{h}_{d}^{-}, \quad h_{u}^{0}=q \sqrt{\frac{n_{u}}{n_{d}}} \bar{h}_{d}^{0} .
$$


Now $h_{u}^{\dagger} h_{d}=\bar{h}_{u}^{+} h_{d}^{0}+\bar{h}_{u}^{0} h_{d}^{-}=q \sqrt{n_{u} / n_{d}}\left(-h_{d}^{-} h_{d}^{0}+h_{d}^{-} h_{d}^{0}\right)=0, h_{u} h_{d}=-q \sqrt{n_{u} / n_{d}} h_{d}^{\dagger} h_{d} \equiv$ $-\sqrt{n_{d} / n_{u}} h^{\dagger} h / q$ and the two Higgs doublet potential $V_{2 h}$ (4.5) reduces to the one Higgs doublet potential,

$$
V_{h}=-2 m_{s}^{2} \frac{\gamma}{q} \sqrt{\frac{n_{d}}{n_{u}}} h^{\dagger} h+\frac{\gamma^{2}}{q^{2}} \frac{n_{d}}{n_{u}}\left(h^{\dagger} h\right)^{2}+\frac{g^{2}+g^{\prime 2}}{8} \frac{\left(q^{2}-1\right)^{2}}{q^{4} n_{u}^{2}}\left(h^{\dagger} h\right)^{2},
$$

where the last term in $V_{2 h}(4.5)$ has dropped out. In this case, $V_{D, H}>0$, which in general violates condition (1.1) unless $q=1$. After SSB, instead of (4.6), one is sitting at

$$
V_{h, \min }^{(q)}=-\frac{m_{s}^{4}}{1+\frac{g^{2}+g^{\prime 2}}{8 \gamma^{2} q^{2} n_{u} n_{d}}\left(q^{2}-1\right)^{2}}
$$

implying $(\Delta V)_{\min }>0$ and condition (1.1) is not satisfied unless $q=1$, when $V_{h \text {, min }}^{(q)}$ is lowest. That is, constraint (2.4c) is energetically preferred, i.e., dynamically determined.

With constraint (2.4c), or $q=1$ for (4.7), the $V_{H, D}$ term in (4.8) drops out, and the Higgs potential for a single Higgs doublet becomes

$$
V_{h}=-2 \kappa m_{s}^{2} h^{\dagger} h+\kappa^{2}\left(h^{\dagger} h\right)^{2}
$$

where $\kappa=\gamma \sqrt{n_{d} / n_{u}}$ is taken to be a real positive (dimensionless) coupling. Since the constraint (2.4c) removes $V_{H, D}$ in (4.5), the gauge couplings do not enter $V_{h}$. This is exactly the SM Higgs potential and its contribution comes entirely from $V_{X} \propto\left|F^{X}\right|^{2}$, where $F^{X}$ here is the vacuum expectation value of the auxilliary field; so $V_{X}=m_{s}^{4}+V_{h}=\left|m_{s}^{2}-\kappa h^{\dagger} h\right|^{2}$, as shown in (1.2). Note that the quartic coupling $\kappa^{2}>0$.

After SSB, the Higgs field acquires a vev, $\left\langle h^{\dagger} h\right\rangle=v^{2} / 2=m_{s}^{2} / \kappa$. Expanding around this minimum, physical massive Higgs boson and the three massless Goldstone bosons emerge, as expected,

$$
m_{h}^{2}=4 m_{s}^{2} \kappa, \quad m_{G^{0}}^{2}=m_{G^{ \pm}}^{2}=0 .
$$

Putting in the observed $m_{H}=125 \mathrm{GeV}$ and $v=246 \mathrm{GeV}$, one finds $\kappa=0.36$ and $m_{s}=$ 104.3 GeV. The triple and quartic Higgs self-couplings are the same as those in SM. The potential value for $V_{X}(1.2)$ after SSB is $V_{X, \min }=0$, irrespective of the values of $m_{s}^{2}$ and the real positive $\kappa$; with

$$
\Delta V=V_{F, H}+V_{H, D}+V_{X}=V_{X},
$$

we now have $(\Delta V)_{\min }=V_{X \text {, min }}=0$, satisfying the condition (1.1) without fine-tuning.

Note that the (warped) SUSY breaking $\overline{\mathrm{D} 3}$-brane tension is completely screened by the Higgs potential, so $\overline{\mathrm{D} 3}$-brane(s) do not break SUSY in this model. SUSY breaking and the uplift from AdS to dS vacuum comes from the $\xi$ term only. However, the SUSY breaking by the Kähler uplift is very small $\left(m_{3 / 2}^{2} \sim \mathcal{G}\left(\Lambda / M_{\mathrm{Pl}}^{2}\right)\right)$, so any SUSY pair will have almost degenerate masses, which is a problem as no superpartner has been observed. Again, this phenomenological problem can be easily solved by imposing the constraints $(2.4 \mathrm{~d})$, which removes all the scalar quarks and scalar leptons as well as the gauginos. Constraint (2.4b) projects out the Higgsinos while constraint (2.4c) reduces the two Higgs doublets to a single doublet, and implies the same Yukawa couplings as those in SM. 


\section{Summary}

The mNSSM model is given by (2.1) together with (2.4). After imposing the constraints (2.4), the particle spectrum of the mNSSM model is reduced to exactly that in the SM (in energy scales around or below $m_{\mathrm{EW}}$ ), except for the very light modes of $S$, $U_{i}$ and $T$ (and the graviton-gravitino pair). Since these modes are not confined to the $\overline{\mathrm{D} 3}$-brane(s), their superpartners (dilatino, the Uenos and the Tino) cannot be projected out by $X$. Here, the role of $\overline{\mathrm{D} 3}$-brane(s) is not to break SUSY (as opposed to the KKLT model [3]), but to cancel the electroweak Higgs potential contribution to $\Lambda$.

Once the contributions to $\Lambda$ from the $\overline{\mathrm{D} 3}$-brane tension cancels the Higgs potential $V_{h, \min }$, the value of $\Lambda$ follows from the $T$ stabilization in the RKU model $[1,4]$. There, the divergent behavior of the probability distribution $P(\Lambda) \sim \Lambda^{-1+k}$ around $\Lambda \sim 0^{+}$follows when we scan over the ratio $z=A / B$ of the coefficients of the two non-perturbative terms in $W_{\mathrm{np}}=A e^{-a T}+B e^{-b T}(2.1)$, while the value of $1 \gg k>0$ depends on the ratio $\beta=b / a$ (as we scan over $a$ and $b$ ). Here the gravitino gains a tiny mass from the $\xi$-induced SUSY breaking, which is for the whole orientifold, not limited to the $\overline{\mathrm{D} 3}$-branes or the throat we live in.

\section{Comments}

Some comments are in order here.

- The (necessary in MSSM) SUSY breaking scale $m_{s}^{4} \gtrsim m_{\mathrm{EW}}^{4}$ are many orders of magnitude bigger than the observed $\Lambda$. This SUSY breaking will generate a huge contribution to $\Lambda$ in the absence of fine-tuning. Therefore, it is neat to see that it is precisely screened by the Higgs potential contribution to $\Lambda$. This cancellation without fine-tuning is probably a necessary condition in any model for a naturally small $\Lambda$.

- In fitting experimental data, the couplings and masses are taken to have physical (i.e., renormalized) values, as measured at the electroweak energy scale, à la the Wilsonian approach. It is important to study the radiative corrections of the model. It is argued that the statistical approach in the RKU model does not have the usual radiative instability problem [40]. Intuitively, this should not be too surprising. To determine $P(\Lambda)$, one scans over ranges of all the parameters of the model that yield a local minimum solution; typical radiative corrections contribute tiny shifts when compared to the variations of the parameters that are already included in the scanning.

- Looking back at the KPV picture [15], where a small stack of $\overline{\mathrm{D} 3}$-branes sits in a "false" vacuum, we see that the electroweak contribution, by lowering the vacuum energy of the "false" vacuum, tends to suppress the tunneling probability when compared to that in the KPV scenario.

- In the model, one expects Kaluza-Klein modes as well as string excitation modes to be present at scales not too far from $m_{\mathrm{EW}}$. Their detection will provide strong 
evidence for the scenario. Furthermore, their spectra will reveal valuable details of the warped throat and exactly where and how the SM sits in the throat.

- The mNSSM has no particle that is a suitable candidate for cold dark matter. However, it has very light scalar fields like the $U_{i}, S$ and $T$ [32, 34, 40], one (or some) of which can play the role of super-light bosonic particles in the fuzzy dark matter scenario [41, 42].

- The model can easily accommodate the $D 3-\overline{\mathrm{D} 3}$ inflationary model, à la the KKLMMT scenario [16]. This scenario should happen in the early universe in another (less warped) throat in the orientifold.

- In the model, we introduce degrees of freedom and terms in the Higgs potential to be projected out later. It will be interesting to find a more direct formulation without going through this seemingly tortuous path.

- We hope the results here, may be together with the speculation on the quark and lepton mass distributions [43], provide hints in the search for the SM in Type IIB string theory and/or F theory.

\section{Acknowledgments}

We thank Cliff Burgess, Keith Dienes, Renata Kallosh, Ling Feng Li, Tao Liu, Liam McAllister, Fernando Quevedo, Gary Shiu, Timm Wrase and Sam Wong for valuable communications and comments. This work is supported in part by the AOE grant AoE/P-404/18-6 issued by the Research Grants Council (RGC) of the Government of the Hong Kong SAR China.

Open Access. This article is distributed under the terms of the Creative Commons Attribution License (CC-BY 4.0), which permits any use, distribution and reproduction in any medium, provided the original author(s) and source are credited.

\section{References}

[1] Y. Sumitomo, S.H.H. Tye and S.S.C. Wong, Statistical distribution of the vacuum energy density in racetrack Kähler uplift models in string theory, JHEP 07 (2013) 052 [arXiv: 1305.0753] [INSPIRE].

[2] S. Andriolo, S.Y. Li and S.H.H. Tye, The cosmological constant and the electroweak scale, JHEP 10 (2019) 212 [arXiv:1812.04873] [INSPIRE].

[3] S. Kachru, R. Kallosh, A.D. Linde and S.P. Trivedi, De Sitter vacua in string theory, Phys. Rev. D 68 (2003) 046005 [hep-th/0301240] [INSPIRE].

[4] Y.-C. Qiu and S.H.H. Tye, Linking the supersymmetric standard model to the cosmological constant, JHEP 01 (2021) 117 [arXiv:2006.16620] [INSPIRE].

[5] M.R. Douglas and S. Kachru, Flux compactification, Rev. Mod. Phys. 79 (2007) 733 [hep-th/0610102] [INSPIRE]. 
[6] K. Becker, M. Becker and J. Schwarz, String theory and M-theory: a modern introduction, Cambridge University Press, Cmabridge U.K. (2006).

[7] L.E. Ibanez and A.M. Uranga, String theory and particle physics: an introduction to string phenomenology, Cambridge University Press, Cambridge U.K. (2012).

[8] D. Baumann and L. McAllister, Inflation and string theory, Cambridge Monographs on Mathematical Physics, Cambridge University Press, Cambridge U.K. (2015) [arXiv: 1404.2601] [INSPIRE].

[9] J.F.G. Cascales, M.P. Garcia del Moral, F. Quevedo and A.M. Uranga, Realistic D-brane models on warped throats: fluxes, hierarchies and moduli stabilization, JHEP 02 (2004) 031 [hep-th/0312051] [INSPIRE].

[10] I. Antoniadis, E. Dudas, D.M. Ghilencea and P. Tziveloglou, Non-linear MSSM, Nucl. Phys. $B 841$ (2010) 157 [arXiv: 1006.1662] [InSPIRE].

[11] R. Kallosh, F. Quevedo and A.M. Uranga, String theory realizations of the nilpotent goldstino, JHEP 12 (2015) 039 [arXiv:1507.07556] [INSPIRE].

[12] M.P. Garcia del Moral, S. Parameswaran, N. Quiroz and I. Zavala, Anti-D3 branes and moduli in non-linear supergravity, JHEP 10 (2017) 185 [arXiv:1707.07059] [INSPIRE].

[13] N. Cribiori, C. Roupec, T. Wrase and Y. Yamada, Supersymmetric anti-D3-brane action in the Kachru-Kallosh-Linde-Trivedi setup, Phys. Rev. D 100 (2019) 066001 [arXiv: 1906.07727] [INSPIRE].

[14] S. Parameswaran and F. Tonioni, Non-supersymmetric string models from Anti-D3-/D7-branes in strongly warped throats, JHEP 12 (2020) 174 [arXiv:2007.11333] [INSPIRE].

[15] S. Kachru, J. Pearson and H.L. Verlinde, Brane/flux annihilation and the string dual of a nonsupersymmetric field theory, JHEP 06 (2002) 021 [hep-th/0112197] [INSPIRE].

[16] S. Kachru, R. Kallosh, A.D. Linde, J.M. Maldacena, L.P. McAllister and S.P. Trivedi, Towards inflation in string theory, JCAP 10 (2003) 013 [hep-th/0308055] [INSPIRE].

[17] P. McGuirk, G. Shiu and F. Ye, Soft branes in supersymmetry-breaking backgrounds, JHEP 07 (2012) 188 [arXiv: 1206.0754] [INSPIRE].

[18] D.V. Volkov and V.P. Akulov, Is the neutrino a goldstone particle?, Phys. Lett. B 46 (1973) 109 [INSPIRE].

[19] M. Roček, Linearizing the Volkov-Akulov model, Phys. Rev. Lett. 41 (1978) 451 [INSPIRE].

[20] E.A. Ivanov and A.A. Kapustnikov, General relationship between linear and nonlinear realizations of supersymmetry, J. Phys. A 11 (1978) 2375 [InSPIRE].

[21] S. Ferrara, R. Kallosh and A. Linde, Cosmology with nilpotent superfields, JHEP 10 (2014) 143 [arXiv: 1408.4096] [INSPIRE].

[22] R. Kallosh and T. Wrase, Emergence of spontaneously broken supersymmetry on an anti-D3-brane in KKLT dS vacua, JHEP 12 (2014) 117 [arXiv:1411.1121] [INSPIRE].

[23] E.A. Bergshoeff, K. Dasgupta, R. Kallosh, A. Van Proeyen and T. Wrase, $\overline{D 3}$ and $d S, J H E P$ 05 (2015) 058 [arXiv: 1502.07627] [INSPIRE].

[24] S.P. Martin, A supersymmetry primer, Adv. Ser. Direct. High Energy Phys. 18 (1998) 1 [hep-ph/9709356] [INSPIRE]. 
[25] U. Lindström and M. Roček, Constrained local superfields, Phys. Rev. D 19 (1979) 2300 [INSPIRE].

[26] Z. Komargodski and N. Seiberg, From linear SUSY to constrained superfields, JHEP 09 (2009) 066 [arXiv:0907.2441] [INSPIRE].

[27] G. Dall'Agata and F. Farakos, Constrained superfields in supergravity, JHEP 02 (2016) 101 [arXiv: 1512.02158] [INSPIRE].

[28] S.B. Giddings, S. Kachru and J. Polchinski, Hierarchies from fluxes in string compactifications, Phys. Rev. D 66 (2002) 106006 [hep-th/0105097] [INSPIRE].

[29] K. Becker, M. Becker, M. Haack and J. Louis, Supersymmetry breaking and alpha-prime corrections to flux induced potentials, JHEP 06 (2002) 060 [hep-th/0204254] [INSPIRE].

[30] V. Balasubramanian and P. Berglund, Stringy corrections to Kähler potentials, SUSY breaking, and the cosmological constant problem, JHEP 11 (2004) 085 [hep-th/0408054] [INSPIRE].

[31] A. Westphal, De Sitter string vacua from Kähler uplifting, JHEP 03 (2007) 102 [hep-th/0611332] [INSPIRE].

[32] M. Rummel and A. Westphal, A sufficient condition for de Sitter vacua in type IIB string theory, JHEP 01 (2012) 020 [arXiv:1107.2115] [INSPIRE].

[33] S. de Alwis and K. Givens, Physical vacua in IIB compactifications with a single Kähler modulus, JHEP 10 (2011) 109 [arXiv:1106.0759] [INSPIRE].

[34] Y. Sumitomo and S.H.H. Tye, A stringy mechanism for a small cosmological constant Multi-moduli cases, JCAP 02 (2013) 006 [arXiv: 1209.5086] [INSPIRE].

[35] J. Louis, M. Rummel, R. Valandro and A. Westphal, Building an explicit de Sitter, JHEP 10 (2012) 163 [arXiv:1208.3208] [INSPIRE].

[36] G. Dall'Agata, E. Dudas and F. Farakos, On the origin of constrained superfields, JHEP 05 (2016) 041 [arXiv: 1603.03416] [InSPIRE].

[37] B. Vercnocke and T. Wrase, Constrained superfields from an anti-D3-brane in KKLT, JHEP 08 (2016) 132 [arXiv: 1605.03961] [INSPIRE].

[38] R. Kallosh, B. Vercnocke and T. Wrase, String theory origin of constrained multiplets, JHEP 09 (2016) 063 [arXiv: 1606.09245] [INSPIRE].

[39] J. Wess and J. Bagger, Supersymmetry and supergravity, Princeton University Press, Princeton U.S.A. (1992).

[40] S.H.H. Tye and S.S.C. Wong, Linking light scalar modes with a small positive cosmological constant in string theory, JHEP 06 (2017) 094 [arXiv: 1611.05786] [INSPIRE].

[41] W. Hu, R. Barkana and A. Gruzinov, Cold and fuzzy dark matter, Phys. Rev. Lett. 85 (2000) 1158 [astro-ph/0003365] [INSPIRE].

[42] D.J.E. Marsh, Axion cosmology, Phys. Rept. 643 (2016) 1 [arXiv:1510.07633] [INSPIRE].

[43] S. Andriolo, S.Y. Li and S.H.H. Tye, String landscape and Fermion masses, Phys. Rev. D $101(2020) 066005$ [arXiv: 1902.06608] [INSPIRE]. 